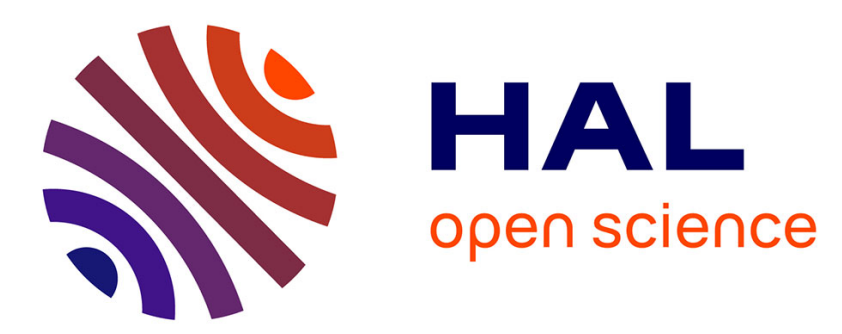

\title{
Validation of 3D spino-pelvic muscle reconstructions based on dedicated MRI sequences for fat-water quantification
}

\author{
Bertrand Moal, J-G Raya, Erwan Jolivet, F. Schwab, B. Blondel, Virginie \\ Lafage, Wafa Skalli
}

\section{To cite this version:}

Bertrand Moal, J-G Raya, Erwan Jolivet, F. Schwab, B. Blondel, et al.. Validation of 3D spino-pelvic muscle reconstructions based on dedicated MRI sequences for fat-water quantification. Innovation and Research in BioMedical engineering, 2014, 35, pp.119-127. 10.1016/j.irbm.2013.12.011 . hal01088726

\section{HAL Id: hal-01088726 https://hal.science/hal-01088726}

Submitted on 13 Jan 2015

HAL is a multi-disciplinary open access archive for the deposit and dissemination of scientific research documents, whether they are published or not. The documents may come from teaching and research institutions in France or abroad, or from public or private research centers.
L'archive ouverte pluridisciplinaire HAL, est destinée au dépôt et à la diffusion de documents scientifiques de niveau recherche, publiés ou non, émanant des établissements d'enseignement et de recherche français ou étrangers, des laboratoires publics ou privés. 


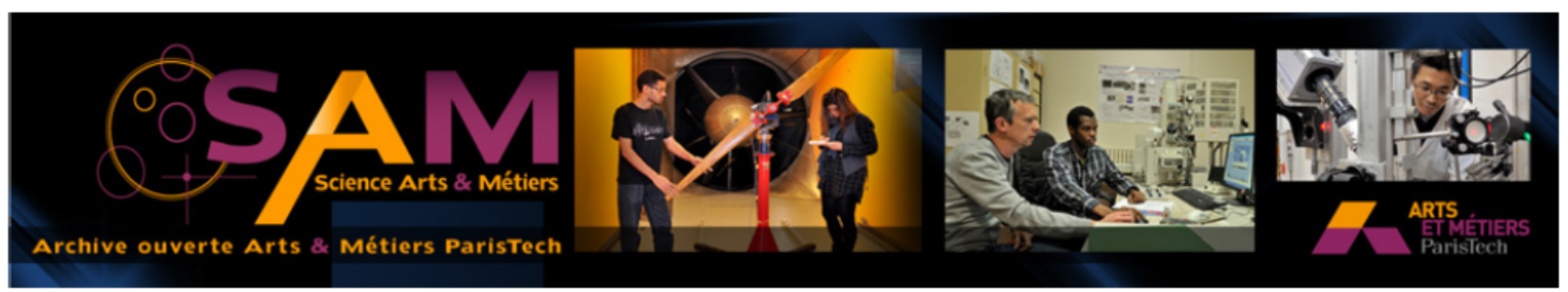

Science Arts \& Métiers (SAM)

is an open access repository that collects the work of Arts et Métiers ParisTech researchers and makes it freely available over the web where possible.

This is an author-deposited version published in: http://sam.ensam.eu Handle ID: .http://hdl.handle.net/10985/9014

To cite this version :

Bertrand MOAL, Jose G. RAYA, Erwan JOLIVET, Franck SCHWAB, Benjamin BLONDEL, Virginie LAFAGE, Wafa SKALLI - Validation of 3D spino-pelvic muscle reconstructions based on dedicated MRI sequences for fat-water quantification - IRBM - Vol. 35, p.119-127 - 2014 


\title{
Validation of 3D spino-pelvic muscle reconstructions based on dedicated MRI sequences for fat-water quantification
}

\author{
B. Moal ${ }^{\text {a,c }}$, J.G. Raya ${ }^{\text {b }}$, E. Jolivet ${ }^{\text {c }}$, F. Schwab ${ }^{\text {a }}$, B. Blondel ${ }^{\text {a }}$, V. Lafage ${ }^{\text {a, } * \text {, W. Skalli }}{ }^{\mathrm{c}}$ \\ ${ }^{a}$ Spine Division, NYU Hospital for Joint Diseases, New York University Langone Medical Center, 306 E. 15th St., Suite 1F, New York, NY, 10003 USA \\ ${ }^{\mathrm{b}}$ Center for Biomedical Imaging, Department of Radiology, New York University Langone Medical Center, 550, First Avenue, New York, NY, 10016 USA \\ ${ }^{\mathrm{c}}$ Laboratoire de biomécanique, arts et métiers ParisTech, 151, boulevard de l'Hôpital, 75013 Paris, France
}

\begin{abstract}
Objectives. - To evaluate a protocol, including MRI acquisition with dedicated sequences for fat-water quantification and semi-automatic segmentation, for 3D geometry measurement and fat infiltration of key muscles of the spino-pelvic complex.

Materials and methods. - MRI protocol: two axial acquisitions from the thoraco-lumbar region to the patella were obtained: one T1-weighted and one based on the Dixon method, permitted to evaluate the proportion of fat inside each muscle. Muscle reconstruction: with Muscl'X software, 3D reconstructions of 18 muscles or groups of muscles were obtained identifying their contours on a limited number of axial images 3D references were obtained only on T1 acquisitions identifying the contour of the muscles on all axial images. Evaluation: for two volunteers, three operators completed reconstructions three times across three sessions. Each reconstruction was projected on the reference to calculate the 'point to surface' error. Mean and maximal axial section, muscle volume, and muscle length calculated from the reconstructions were compared to reference values, and intra- and inter-operator variability for those parameters were evaluated.

Results. - 2xRMS 'point to surface' error was below $3 \mathrm{~mm}$, on average. The agreement between the two methods was variable between muscles $[-4.50 ; 8.00 \%]$ for the mean axial section, the length and the volume. Intra- and inter-operator variability were less than $5 \%$ and comparison of variability for the Fat and $\mathrm{T} 1$ reconstructions did not reveal any significant differences.

Discussion. - Excellent inter- and intra-operator reliability was demonstrated for 3D muscular reconstruction using the DPSO method and Dixon images that allowed generation of patient-specific musculoskeletal models.
\end{abstract}

\section{Introduction}

The muscular system plays an essential role in the maintenance of postural balance; however, in clinical practice as well as research, investigation into the relationship between the muscular system and postural pathologies such as adult spinal deformity, has been limited.

The current lack of knowledge related to soft tissue stabilizers may be attributed to the absence of a relevant tool to evaluate the muscular system as a whole. The large number of muscles involved in postural maintenance makes a global analysis difficult and time consuming. In the case of adult spinal deformity (ASD), recent research has highlighted the critical role of sagittal spino-pelvic alignment in patient-reported pain and disability

\footnotetext{
* Corresponding author.

E-mail address: virginie.lafage @gmail.com (V. Lafage).
}

[1-5]. Therefore, key muscles involved in pelvic-positioning and lumbar-stabilization are at the forefront of research needs.

Preliminary efforts have been directed towards understanding the muscular envelope of the spine using histological analyses [6-9], measurement of muscular strength [10-12], and measurement of electromyographical signals $[13,14]$. However, these approaches are not adapted for study of a large number of muscle groups. Other studies, using imaging such as MRI or CT scan, correlated measurement of the muscle cross-sectional areas (via ultrasound, CT-scan or MRI) [15-23], or measurement of muscular density (via CT-scan or MRI) [10-12,17-21,23-25], with chronic back pain or spinal surgery outcome. The limitations of these approaches lie in the difficulty to represent variability in volume of an entire muscle [26].

To overcome these limitations, Jolivet et al. [27,28] developed a method of three-dimensional muscle reconstruction via segmentation of a small number of axial images (MRI or CTscan). This method, based on the deformation of a parametric 


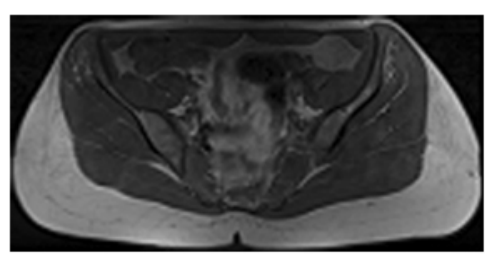

T1 image

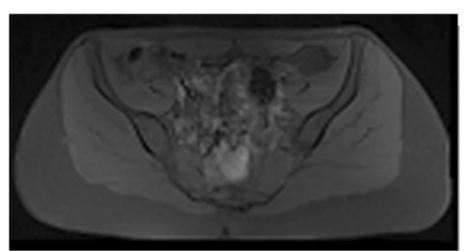

Water image

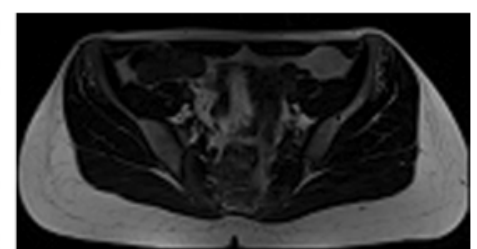

Fat image

Fig. 1. Examples of a T1 (left), water (center), and Fat (right) image.

specific object(DPSO), has been successfully implemented with CT scans for analysis of muscles involved in knee motion [29] and muscle groups around the hip joint [28]. The CT-scan modality presents two advantages: good contrast quality for muscle segmentation and good reliability in terms of fat and muscle density. CT scans allow for both a reproducible analysis of muscle geometry and a quantified evaluation of fat infiltration [28]. Nevertheless, the radiation exposure from CT scans renders it unacceptable as a tool for studies involving ASD patients who are already frequently subjected to radiographic examination. Notably, the DPSO method has also been performed using MRI T1 sequences [29]. However, the inhomogeneity in the magnetic field applied did not allow an accurate quantification of the fat infiltration (without fat infiltration, the muscle volume is an incomplete descriptor).

In order to avoid the problem of inhomogeneity and obtain an accurate quantification of fat infiltration, Dixon et al. [30] developed a specific acquisition sequence where two images are obtained: one in which the intensity of each voxel is correlated with the quantity of fat and the other in which the intensity of each voxel is correlated with the quantity of water. This method was then improved by Glover et al. [31]. To our knowledge, the feasibility of 3D muscular reconstruction on MRI with the Dixon method was not studied.

The objective of this study was to evaluate the feasibility of an MRI protocol with dedicated sequences for fat-water quantification to assess the 3D geometry and homogeneity (fat infiltration) of key muscles in the spino-pelvic complex.

\section{Methods}

\subsection{Subject sample}

Two asymptomatic female adult volunteers were included in this pilot study: volunteer A (35 years, $68 \mathrm{~kg}$ ) and volunteer B (38 years, $91 \mathrm{~kg}$ ).

\subsection{MRI acquisition}

MRI was performed on a $3 \mathrm{~T}$ whole-body scanner (Magnetom Verio, Siemens Healthcare, Erlangen, Germany) using a 24-channel spine matrix coil and three 4-channel flex coils from the same vendor. The imaging protocol included a T1-weighted turbo spin-echo (T1 TSE) sequence $(\mathrm{TR} / \mathrm{TE}=1220 / 11 \mathrm{~ms}$, acquisition matrix $=512 \times 384$, in plane resolution $=0.98 \times 0.98 \mathrm{~mm}^{2}$, slice thickness $=5 \mathrm{~mm}$, slice gap $=5 \mathrm{~mm}$, parallel imaging acceleration factor (iPat) $=2$, 40 slices, flip angle $=150^{\circ}$, bandwidth $=219 \mathrm{~Hz} /$ pixel, turbo factor $=5$, acquisition time $=2: 15 \mathrm{~min} / 40$ slices, 160 slices by patients) and a T1-weighted TSE sequence for applying the three point Dixon method [30-33] (TR/TE $=829 / 15.7 \mathrm{~ms}$, acquisition matrix $=512 \times 384$, in plane resolution $=0.98 \times 0.98 \mathrm{~mm}^{2}$, slice thickness $=5 \mathrm{~mm}$, slice gap $=5 \mathrm{~mm}$, iPat $=2,40$ slices, flip angle $=150^{\circ}$, bandwidth $=315 \mathrm{~Hz} /$ pixel, turbo factor $=3$, echo spacing $=15.7$, acquisition time $=4: 38 \mathrm{~min} / 40$ slices, 160 slices by patients). Water and Fat images were automatically generated by the scanner from the TSE images for the three point Dixon method (Fig. 1). Both sequences had exactly the same slice position and orientation. Image volume covered the proximal tibia to the lumbar spine (Th12 vertebra) and was acquired in three stages. Total acquisition time was $45 \mathrm{~min}$.

\section{3. $3 D$ muscle reconstruction (DPSO method)}

The 3D reconstruction of individual muscles was performed using Muscl'X software, a custom software, (Laboratory of Biomechanics, Arts et Métiers ParisTech, France). Using the axial MR images, the software generated the 3D geometry of each muscle. The reconstruction technique was based on the DPSO algorithm as described in the literature [27-29] and briefly summarized hereafter (Fig. 2).

For each muscle, a subset of MRI axial slices (MSS: manually segmented slices) were manually segmented. The optimal percentage of MSS (defined as the number of MSS divided by the total number of slices covering the entire muscle) of each muscle is reported in Table 1 [34]. Of note, complex muscle geometries required a larger percentage of MSS than simple geometries. Using contrast differences, these manual segmentations were then optimized (Fig. 2a). The contours were then approximated by ellipses (Fig. 2b) and cubic spline interpolation was used to interpolate ellipses in all non-outlined slices covering the muscle (Fig. 2c). These interpolated ellipses generated a 3D parametric object (Fig. 2d). Finally, using a kriging algorithm [35], the parametric object was deformed non-linearly using the manual segmentations of MSS as control points (Fig. 2e). Contrast enhancements were used to optimize the segmentation at each slice. Once all muscles were reconstructed, a geometry-correction algorithm was applied to eliminate muscle segmentation interpenetration and a visual verification was performed to correct local geometric deformities. Finally, 3D meshed reconstructions of each muscle were obtained. 

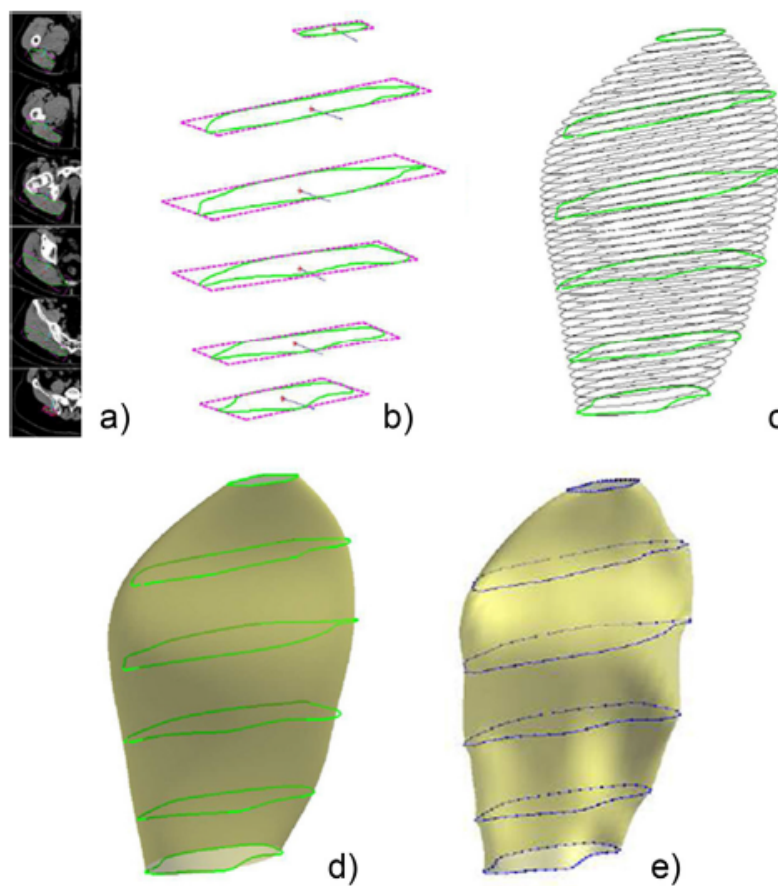

c)

e)

Fig. 2. Overview of the reconstruction method [28]: a: subset of MRI axial slices with manually segmented axial sections; b: contours approximated by ellipses [represented on the figure by a green rectangle whose length and width correspond to the major and minor axes of the ellipses]; c: cubic spline interpolation used to interpolate ellipses in all non-outlined slices covering the muscle; $d$ : interpolated ellipses generated a 3D parametric object; e: non-linear deformation of the parametric object was deformed using the manual segmentations.

\subsection{Muscles of interest}

Table 1 describes the right- and left-sided muscles or groups of muscles analyzed in this study. Muscles were chosen based on their potential role in regulating the position of the pelvis and the spine. Because the delineation of certain muscles was difficult and in order to decrease the number of reconstructed muscles,

Table 1

Muscles analyzed in this study, and optimal percentage of MSS slices.

\begin{tabular}{ll}
\hline Muscle reconstructed(right and left) & Percentage of MSS \\
\hline Adductor & 20 \\
Biceps femoris & 12 \\
Erector spinae & 15 \\
Gluteus maximus & 18 \\
Gluteus medius & 25 \\
Gluteus minimus & 30 \\
Gracilis & 10 \\
Iliacus & 25 \\
Obliquus & 20 \\
Psoas & 10 \\
Quadratus lumborum & 18 \\
Rectus abdominus & 12 \\
Rectus femoris & 13 \\
Sartorius & 10 \\
Semi-membranous tendinosis & 11 \\
Tensor Fascia Lata & 15 \\
Vastus lateralis inter & 15 \\
Vastus medialis & 15 \\
\hline
\end{tabular}

MSS: manually segmented slices. some of the muscles were regrouped. The adductor longus, brevis and magnus were reconstructed into a single group named "Adductor". In addition, the transversus abdominis muscle, internus obliquus, and externus obliquus were considered as a single group named "Obliquus" (they were reconstructed from their caudal insertion up to the liver). The rectus abdominus, the psoas, and the erector spinae were reconstructed from their caudal insertion to the superior endplate of the first lumbar vertebra. The short- and long- heads of the biceps femoris were grouped together ("Biceps femoris"), as were the semimembranosus and semitendinosus muscles ("Semi-membranous tendinosus"), and the vastus lateralis and vastus intermedius muscles ("vastus lateralis inter"). In total, 18 muscles, right and left, were analyzed.

\subsection{MR parameters}

Based on 3D reconstructions, the following parameters were calculated for each muscle: mean and maximal axial section (AS), muscle volume, and muscle length. Muscle length was defined as the sum of distances between barycenters of all consecutives slices.

From the Water and Fat images, the relative fat content for each voxel, fat/water ratio, was calculated using Eq. (1).

Fat/Water Ratio $=100 \times \frac{\mathrm{SI}_{\text {fat }}}{\mathrm{SI}_{\text {fat }}+\mathrm{SI}_{\text {water }}}$

where $\mathrm{SI}_{\text {fat }}$ represents the signal intensity of the Fat image and $\mathrm{SI}_{\text {water }}$ represents the signal intensity of the Water image. For each muscle, all voxels contained in the muscle outline were identified. The average fat/water ratio was calculated over all voxels contained in each muscle allowing a quantification of the fat inside each muscle.

\subsection{Evaluation of the protocol}

All the operators involved in the protocol evaluation were experienced in reading muscular anatomy on MR images and received training in the use of the software (Muscl'X). For the DPSO method, the first and last slices of each muscle were manually segmented and used as limits for all reconstructions; all operators used exactly the same number of MSS (Table 1) while the selection of the actual MSS was left to the operator's discretion.

\subsection{Evaluation of the DPSO method}

For each muscle of the studied volunteers, a reference object (i.e. 3D geometry) was generated on $\mathrm{T} 1$ images by manually contouring all MR axial images covering it. Fig. 3 presents the $3 \mathrm{D}$ reconstruction of volunteer $\mathrm{A}$. This method of reconstruction was named the reference method and the $3 \mathrm{D}$ reconstruction objects were named references. The references for volunteers A and B were obtained by one operator. Using the DPSO method and the number of MSS presented in Table 1, this operator reconstructed once on the Fat images and once on the T1 images all the muscles for both volunteers. 


\section{Volunteer A}

\begin{tabular}{|l|}
\hline Adductor \\
\hline Biceps femoris \\
\hline Erectus spinae \\
Gluteus maximus \\
Gluteus medius \\
Gluteus minimus \\
Gracilis \\
Tliacus \\
Obliquus \\
Psoas \\
Quadratus lumborum \\
Rectus abdominus \\
Rectus femoris \\
Sartorius \\
Semi mem tendi \\
Tensor Fascia Lata \\
Vastus lateralis inter \\
Vastus medialis \\
\hline
\end{tabular}

AnteriorView

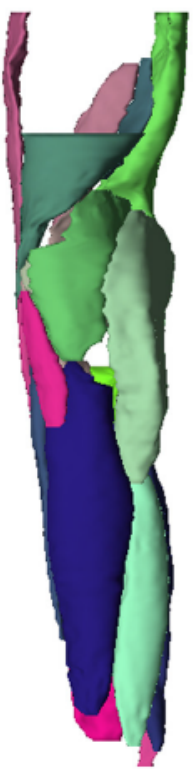

Lateral View
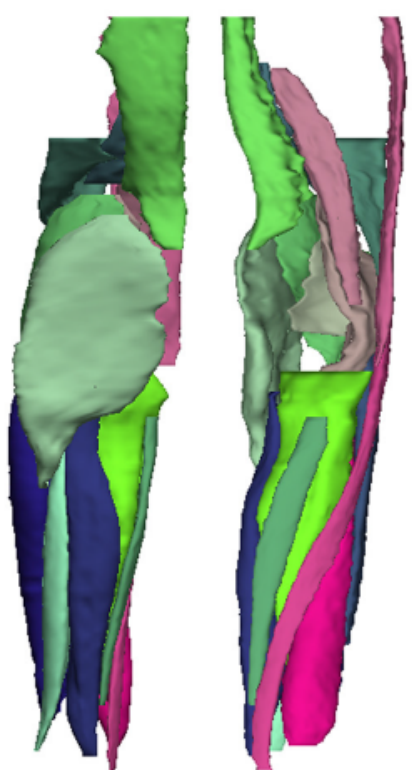

Posterior View Medial View

Fig. 3. Reference reconstruction for volunteer A including only left muscles; anterior, lateral, posterior and medial views.

The agreement between the DPSO with T1 or Fat images and the reference method (T1 images) was assessed with the percentage of difference between the methods [36] for the following parameters: maximal AS, mean AS, length, and volume. The mean, standard deviation (STD), maximum and minimum amount of difference between the reference values and the reconstruction values were expressed as a percentage of the reference values. The limits of the agreement were defined by the interval [Mean $-2 \times$ STD, Mean $+2 \times$ STD] .

\subsection{Reproducibility of the DPSO method for T1 and Fat images}

For this study, the intra- and inter-operator variability of the maximal AS, mean AS, length and volume were calculated in addition to shape accuracy. In this context, three operators using the DPSO methods completed the reconstruction of each muscle for each volunteer three times based on the T1 images ("T1 reconstruction") and three times based on the Fat images ("Fat reconstruction"). Thus, for each muscle of each volunteer, reconstruction was performed nine times for each sequence ( 3 operators $\times 3$ sessions for T1 and Fat images). The muscular reconstructions for each volunteer were repeated with a minimum interval of 3 days for each operator.

\subsubsection{Intra- and inter-variability}

For the maximal AS, mean AS, length and volume, the reliability (intra-operator variability) and the reproducibility (inter-operator variability) from the T1 and Fat reconstructions were computed according to the ISO standard 5725-2:1994 [37] as the coefficient of variation expressed as a percentage. Two different coefficients of variation were calculated: the coefficient of variation of each operator for each volunteer and the coefficient of variation of the average of the three operators for each volunteer. The intra-operator variability was the root mean square of the first coefficient of variation and the interoperator variability was the root mean square of the sum of the two coefficients of variation. The intra- and inter-operator variability for the average fat/water ratio was calculated only using the Fat reconstructions.

\subsubsection{Shape accuracy}

In order to evaluate shape accuracy of the reconstructions, the data obtained from the three operators were compared to the reference previously reconstructed for the evaluation of the DPSO method. Differences in shape between the reference and each reconstruction obtained with the DPSO method (T1 and Fat reconstructions) were evaluated by projecting perpendicularly to the reference in three dimensions the points of the reconstructed muscles onto the reference surface. The $95 \%$ confidence interval for the average point-to-surface-distance [38] was evaluated as two times the root mean square (2xRMS). The mean, standard deviation, minimum and maximum values of the $2 x$ RMS distances were calculated separately for the $\mathrm{T} 1$ reconstructions and the Fat reconstructions pooling the reconstructions of all operators together.

Statistical analyses were conducted using the software SPSS version 17 (SPSS, Inc, Chicago, IL). Differences between the reproducibility of $\mathrm{T} 1$ and Fat reconstructions were investigated using a two-sided paired $t$-test with a level of significance of 0.05 . 
Table 2

Results for volunteers A and B obtained with reference method (except fat/water ratio: average on all the Fat reconstructions).

\begin{tabular}{|c|c|c|c|c|c|c|c|c|c|c|}
\hline \multirow[t]{2}{*}{ Name } & \multicolumn{2}{|c|}{$\operatorname{Max} \mathrm{AS}\left(\mathrm{mm}^{2}\right)$} & \multicolumn{2}{|c|}{ Mean AS $\left(\mathrm{mm}^{2}\right)$} & \multicolumn{2}{|c|}{ Length (mm) } & \multicolumn{2}{|c|}{ Volume $\left(\mathrm{mm}^{3}\right)$} & \multicolumn{2}{|c|}{ Fat/water ratio $(\%)$} \\
\hline & A & B & A & B & A & B & A & B & A & B \\
\hline Adductor L & 4810 & 5426 & 2845 & 3050 & 300 & 329 & 828738 & 932146 & 12.78 & 20.45 \\
\hline Adductor R & 4865 & 5173 & 2714 & 2990 & 301 & 327 & 776222 & 916293 & 12.45 & 18.11 \\
\hline Biceps femoris $\mathrm{L}$ & 1366 & 1438 & 662 & 705 & 328 & 357 & 213860 & 238231 & 14.03 & 23.33 \\
\hline Biceps femoris $\mathrm{R}$ & 1265 & 1428 & 632 & 717 & 337 & 352 & 207257 & 245682 & 17.76 & 21.74 \\
\hline Erectus spinae L & 2533 & 2768 & 1889 & 1832 & 267 & 212 & 438288 & 341158 & 21.90 & 28.30 \\
\hline Erectus spinae $\mathrm{R}$ & 2714 & 2615 & 1890 & 1830 & 261 & 212 & 439112 & 340376 & 23.27 & 28.49 \\
\hline Gluteus maximus L & 4273 & 5943 & 2690 & 3321 & 300 & 318 & 737806 & 892654 & 18.75 & 33.84 \\
\hline Gluteus maximus $\mathrm{R}$ & 3963 & 5561 & 2571 & 3154 & 289 & 319 & 692013 & 878789 & 19.63 & 33.70 \\
\hline Gluteus medius L & 3054 & 3040 & 1744 & 1846 & 205 & 205 & 303772 & 302643 & 12.41 & 19.27 \\
\hline Gluteus medius R & 2728 & 3040 & 1653 & 1673 & 210 & 206 & 287706 & 298895 & 12.72 & 19.12 \\
\hline Gluteus minimus L & 1362 & 1195 & 943 & 804 & 131 & 116 & 93116 & 70784 & 15.60 & 19.54 \\
\hline Gluteus minimus $\mathbf{R}$ & 1647 & 1161 & 976 & 689 & 133 & 125 & 101465 & 63928 & 16.71 & 18.25 \\
\hline Gracilis L & 331 & 544 & 229 & 351 & 300 & 301 & 65342 & 98798 & 12.05 & 20.59 \\
\hline Gracilis R & 367 & 592 & 245 & 396 & 297 & 288 & 69990 & 107238 & 10.99 & 19.36 \\
\hline Iliacus L & 1317 & 1263 & 877 & 766 & 253 & 246 & 200654 & 163212 & 15.59 & 21.67 \\
\hline Iliacus R & 1336 & 1341 & 858 & 745 & 245 & 242 & 187503 & 158666 & 12.82 & 17.53 \\
\hline Obliquus L & 3046 & 2537 & 1157 & 1228 & 245 & 213 & 234643 & 225859 & 25.85 & 31.44 \\
\hline Obliquus R & 3032 & 2315 & 1156 & 1150 & 263 & 210 & 246104 & 212022 & 25.00 & 33.22 \\
\hline Psoas L & 1325 & 1155 & 721 & 657 & 258 & 237 & 171766 & 146658 & 17.20 & 26.03 \\
\hline Psoas R & 1356 & 1185 & 740 & 654 & 255 & 240 & 176296 & 146012 & 17.03 & 23.91 \\
\hline Quadratus lumborum L & 862 & 536 & 459 & 365 & 153 & 137 & 55572 & 42194 & 18.02 & 29.33 \\
\hline Quadratus lumborum R & 701 & 567 & 347 & 363 & 159 & 139 & 45449 & 41563 & 22.20 & 26.60 \\
\hline Rectus abdominus L & 1069 & 729 & 657 & 612 & 357 & 303 & 226174 & 178018 & 24.54 & 32.28 \\
\hline Rectus abdominus $\mathrm{R}$ & 816 & 798 & 623 & 594 & 359 & 301 & 214962 & 172540 & 22.88 & 31.46 \\
\hline Rectus femoris L & 878 & 1196 & 526 & 700 & 309 & 327 & 161707 & 222483 & 13.20 & 15.60 \\
\hline Rectus femoris $\mathrm{R}$ & 934 & 1075 & 546 & 641 & 304 & 326 & 162332 & 203609 & 10.37 & 15.49 \\
\hline Sartorius L & 330 & 438 & 214 & 308 & 498 & 465 & 99095 & 132455 & 18.25 & 23.86 \\
\hline Sartorius R & 308 & 406 & 234 & 317 & 470 & 468 & 100298 & 134812 & 14.02 & 22.94 \\
\hline Semi mem tendi $\mathrm{L}$ & 1575 & 2416 & 906 & 1343 & 370 & 367 & 328975 & 474250 & 18.78 & 21.68 \\
\hline Semi mem tendi $\mathrm{R}$ & 1425 & 2358 & 906 & 1283 & 372 & 378 & 329319 & 459471 & 16.14 & 21.48 \\
\hline Tensor Fascia Lata L & 596 & 672 & 378 & 425 & 144 & 151 & 52234 & 60726 & 16.78 & 22.75 \\
\hline Tensor Fascia Lata R & 567 & 632 & 369 & 427 & 141 & 148 & 50795 & 58669 & 17.02 & 23.96 \\
\hline Vastus lateralis inter L & 3648 & 4558 & 2288 & 2657 & 371 & 372 & 810584 & 940247 & 11.11 & 16.37 \\
\hline Vastus lateralis inter $\mathrm{R}$ & 3608 & 4675 & 2212 & 2762 & 383 & 372 & 794354 & 964067 & 11.40 & 15.16 \\
\hline Vastus medialis L & 1837 & 2235 & 1003 & 1194 & 354 & 322 & 344227 & 373751 & 10.84 & 15.10 \\
\hline Vastus medialis R & 1598 & 2123 & 956 & 1290 & 331 & 308 & 299664 & 384815 & 9.49 & 13.24 \\
\hline
\end{tabular}

AS: axial section.

\section{Results}

\subsection{Reconstruction time}

The time to obtain a reconstruction (including visual verification to correct local geometric errors) of the muscles for one volunteer was 7 hours. For the reference, the time of reconstruction was between 14 and 15 hours.

\subsection{Individual results per subject}

The reference values for volunteers A and B are presented in Table 2. This table includes results of the maximal AS, mean AS, length, volume and fat/water ratio (average of all Fat reconstructions) for each muscle.

\subsection{Evaluation of the DPSO method}

Table 3 illustrates the agreement between the DPSO methods with Fat and T1 images and the reference methods. The limits of agreement between the DPSO methods with Fat and the reference methods were [-3.50;7.89] for the volume. For both sequences, the maximal AS showed the largest errors. The results demonstrated that for the volume and the mean AS, the agreement between the DPSO method with T1 images and the reference method was better than the agreement between the DPSO method with Fat images and the Reference. On average, the DSPO method, as compared to the reference method, tended to underestimate all parameters (between $0.74 \%$ to $2.20 \%$ ).

\subsection{Intra- and inter-operator variability (DPSO method with $\mathrm{Tl}$ and Fat images only)}

The intra- and inter-operator variability results for the $\mathrm{T} 1$ and Fat reconstructions of each muscle are summarized in terms of mean, standard deviation, maximal and minimal values for all the muscles in Tables 4 and 5. On average, the intra- and interoperator variability was less than $5 \%$ for all parameters. For both T1 and Fat images, only the gluteus minimus (both left and right) 
Variability in percentage between reference methods and DSPO methods for T1 and Fat images.

\begin{tabular}{|c|c|c|c|c|c|c|c|c|}
\hline \multirow[t]{3}{*}{ On all the muscles and both volunteers } & \multicolumn{8}{|c|}{$100 \times($ reference method-DSPO Method)/reference method for one operator and both volunteers } \\
\hline & \multicolumn{2}{|c|}{ Max AS } & \multicolumn{2}{|c|}{ Mean $\mathrm{AS}^{\mathrm{a}}$} & \multicolumn{2}{|c|}{ Length } & \multicolumn{2}{|c|}{ Volume $^{\mathrm{a}}$} \\
\hline & T1 & Fat & T1 & Fat & T1 & Fat & T1 & Fat \\
\hline Mean & 1.21 & 1.16 & 0.74 & 2.20 & 0.91 & 0.44 & 1.10 & 2.19 \\
\hline Std & 4.87 & 6.17 & 2.57 & 2.82 & 1.79 & 2.44 & 2.50 & 2.85 \\
\hline Limits of agreement sup (Mean $+2 \times$ Std) & 10.95 & 13.49 & 5.87 & 7.84 & 4.49 & 5.32 & 6.10 & 7.89 \\
\hline Limits of agreement inf (Mean $-2 \times$ Std) & -8.53 & -11.18 & -4.39 & -3.45 & -2.68 & -4.45 & -3.91 & -3.50 \\
\hline Max & 15.43 & 15.57 & 6.01 & 6.92 & 6.18 & 10.39 & 5.92 & 6.92 \\
\hline Min & -9.41 & -17.75 & -6.22 & -6.50 & -2.96 & -4.08 & -6.62 & -6.46 \\
\hline
\end{tabular}

AS: axial section.

a Significant difference between Fat and T1.

Table 4

Intra-operator variability in percentage (coefficient of variation).

\begin{tabular}{|c|c|c|c|c|c|c|c|c|c|}
\hline & \multicolumn{2}{|c|}{ Max AS } & \multicolumn{2}{|c|}{ Mean AS } & \multicolumn{2}{|c|}{ Length $^{a}$} & \multicolumn{2}{|c|}{ Volume } & \multirow{2}{*}{$\frac{\text { Fat/water ratio }}{\text { Fat }}$} \\
\hline & $\mathrm{T} 1$ & Fat & $\mathrm{T} 1$ & Fat & $\mathrm{T} 1$ & Fat & T1 & Fat & \\
\hline Mean(on all the muscles) & 4.08 & 3.76 & 2.16 & 2.05 & 1.78 & 1.26 & 2.16 & 2.05 & 2.23 \\
\hline Std (on all the muscles) & 2.54 & 2.19 & 0.87 & 1.02 & 2.05 & 1.02 & 0.86 & 1.01 & 1.25 \\
\hline Max (on all the muscles) & 12.49 & 10.66 & 3.81 & 5.60 & 9.93 & 5.16 & 3.75 & 5.62 & 5.56 \\
\hline Min (on all the muscles) & 0.93 & 1.44 & 0.94 & 0.89 & 0.17 & 0.10 & 0.95 & 0.90 & 0.82 \\
\hline
\end{tabular}

AS: axial section.

a Significant difference between Fat and T1.

Table 5

Inter-operator variability in percentage (coefficient of variation).

\begin{tabular}{|c|c|c|c|c|c|c|c|c|c|}
\hline & \multicolumn{2}{|c|}{ Max AS } & \multicolumn{2}{|c|}{ Mean AS } & \multicolumn{2}{|c|}{ Length $^{a}$} & \multicolumn{2}{|c|}{ Volume } & \multirow{2}{*}{$\begin{array}{l}\text { Fat/water ratio } \\
\text { Fat }\end{array}$} \\
\hline & $\mathrm{T} 1$ & Fat & $\mathrm{T} 1$ & Fat & $\mathrm{T} 1$ & Fat & $\mathrm{T} 1$ & Fat & \\
\hline Mean(on all the muscles) & 4.63 & 4.50 & 2.56 & 2.61 & 1.86 & 1.42 & 2.55 & 2.61 & 2.98 \\
\hline Std (on all the muscles) & 3.12 & 3.33 & 1.09 & 1.82 & 2.10 & 1.27 & 1.07 & 1.82 & 1.76 \\
\hline Max (on all the muscles) & 15.65 & 19.62 & 5.80 & 11.67 & 9.93 & 5.93 & 5.78 & 11.80 & 10.59 \\
\hline Min (on all the muscles) & 1.01 & 1.46 & 0.94 & 0.93 & 0.17 & 0.14 & 0.95 & 0.93 & 1.24 \\
\hline
\end{tabular}

AS: axial section.

a Significant difference between Fat and T1.

had an intra-operator variability greater than $5 \%$ for the mean AS, length, and volume.

The other muscles with an inter-operator variability greater than $5 \%$ were the rectus abdominus and the gluteus medius. For the $\mathrm{T} 1$ image, the inter-operator variability of the length was equal to $5.87 \%$ for the gluteus medius right. On T1 images, the rectus abdominus left inter-operator variability was $5.80 \%$ for the mean AS and $5.78 \%$ for the volume.

For the average of fat/water ratio, the left and right gluteus minima and the left vastus medialis were the only muscles with an intra-operator variability greater than $5 \%$. Except for the length, no significant differences were found between $\mathrm{T} 1$ and Fat reconstructions in terms of intra- or inter-operator variability.

\subsection{Shape accuracy analysis}

The results of the point-to-surface-distances for the T1 and Fat reconstructions of each muscle are summarized in terms of mean, standard deviation, and minimal and maximal values for all muscles in Table 6. The mean 2xRMS values were less than $3 \mathrm{~mm}$ (i.e. less than 3 voxels), with significantly smaller values for the $\mathrm{T} 1$ reconstructions than for the Fat reconstructions. The minimum 2xRMS was also significantly smaller for the T1 images. The maximal $2 x R M S$ value in terms of point-to-surfacedistance was less for the $\mathrm{T} 1$ reconstructions $(11.30 \mathrm{~mm})$ than for the Fat reconstructions $(16.41 \mathrm{~mm})$.

\section{Discussion}

To the best of our knowledge, there are only two studies $[39,40]$ which analyze the relationship between posture and the muscular system. Pomero et al. [39,40] used a method, which combined stereo-radiographic $3 \mathrm{D}$ reconstructions of the spine, forceplate acquisition, physical testing (Cybex II), MRI reconstruction, and muscle modeling. While preliminary results indicate that establishing a mechanical model of the spine is 
Distance points surfaces in mm. Mean, Std, Min and Max 2xRMS with the average, standard deviation, minimal and maximal of 2xRMS on all reconstructions of one muscle (9 reconstructions/per muscle).

\begin{tabular}{|c|c|c|c|c|c|c|c|c|}
\hline & \multicolumn{8}{|c|}{ Projection: distance points surfaces in $\mathrm{mm}$} \\
\hline & \multicolumn{2}{|c|}{ Mean $2 x$ MS $^{a}$} & \multicolumn{2}{|c|}{ Std 2xRMS } & \multicolumn{2}{|c|}{ Min $2 x_{R M S}{ }^{a}$} & \multicolumn{2}{|c|}{ Max 2xRMS } \\
\hline & $\mathrm{T} 1$ & Fat & $\mathrm{T} 1$ & Fat & $\mathrm{T} 1$ & Fat & $\mathrm{T} 1$ & Fat \\
\hline Mean & 2.62 & 2.95 & 0.48 & 0.52 & 1.96 & 2.36 & 3.80 & 4.30 \\
\hline Std & 0.73 & 0.91 & 0.35 & 0.53 & 0.57 & 0.63 & 1.85 & 2.72 \\
\hline Min & 1.27 & 1.32 & 0.16 & 0.09 & 0.88 & 1.15 & 1.83 & 1.57 \\
\hline Max & 4.15 & 5.80 & 1.98 & 2.91 & 3.24 & 3.76 & 11.30 & 16.41 \\
\hline
\end{tabular}

a Significant difference between Fat and T1.

possible, this complex approach does not appear to be clinically applicable, and thus carries limited practical value.

As mechanical models and diagnostic protocols can offer significant contributions to the treatment of degenerative pathologies and deformities, the need exists for further research into the role of soft tissue stabilizers in postural balance.

The reproducibility of the DPSO methods with MR images was previously established by Sudhoff et al. [29] for the muscles involved in knee motion. Reconstructions were based on volume interpolated breath-hold examination (VIBE) images. In order to obtain good visualization of soft tissue, the authors used T1 images. The correlation between the intensity of the signal and the quantification of fat was not reliable due to the lack of homogeneity of the magnetic field.

In order to quantify both muscle geometry and fat infiltration, our study utilized the three point Dixon method. This method permits calculation of water and fat images. The method was originally developed by Dixon [30] using a two point (in phase and out of phase) acquisition. The two point Dixon method is sensitive to inhomogeneity of the magnetic field. To avoid this problem, Glover and Schneider introduced the three point Dixon method [31]. As demonstrated by Bley et al. [41], this method is robust and, as for CT analysis, permits separation of the fat and water volumes (Fat and Water images), thus allowing quantification of fat infiltration.

The time needed to complete the 3D reconstruction of all the studied muscles from the thoraco-lumbar region to the patella (Table 1), with a systematic outline of every slice (reference methods), was between 14 and 15 hours, while the time needed to obtain the reconstruction with Muscl'X software and DPSO methods was about 7 hours. The substantial reduction in time renders the software compatible for clinical research but further research should aim at reducing the time needed for reconstruction.

In this study, the reference object was constructed based on T1 images; this sequence was chosen based on the contrast between fat and muscle and for its wide use in clinical applications. The agreement between the two methods (T1 and Fat images) was variable between muscles and comprised between $-4.50 \%$ and $+8.00 \%$ for the mean AS, the length and the volume. These values of agreement reflect two study volunteers and analysis of 36 muscles. For the same muscle, it appeared that on average the volume and the mean AS obtained with the DPSO method was smaller (T1: $\sim 1 \%$, Fat: $\sim 2 \%$ ) than the volume obtained with the reference method. The difference was significantly greater for the DPSO method with Fat image. This systematic bias could be explained by the use of the contrast optimization which can reduce the AS of the non-manually segmented slices. However, this error did not hamper the use of the DPSO method in the 3D geometry assessment of the muscle.

The segmentation of the three point Dixon method had the same quality of segmentation as the segmentation on T1 images. The Fat images had higher fat/muscle contrast than the T1 images; however, visibility of the epimysium and the bone/muscle contrast were better delineated in the T1 images (Fig. 4). This may also explain why the systematic error in the agreement between methods was greater for DPSO with Fat images than for DPSO with T1 images.

An analysis of the accuracy of muscle shape (i.e. comparison of T1/Fat reconstructions with the reference) revealed small point-to-surface-distance errors (mean 2xRMS errors of $2.6 \mathrm{~mm}$ and $2.95 \mathrm{~mm}$ for $\mathrm{T} 1$ and Fat reconstructions, respectively). The references were only constructed on the T1 image, which could explain a greater difference in terms of point to surface on the Fat images. The DPSO method leads to error when a muscle is poorly approximated by an ellipse (e.g. the adductor), or when there are abrupt variations in muscle shape between adjacent slices (e.g. the iliacus). However, errors in terms of shape had little impact on the agreement for other parameters: muscle length, mean AS, and volumes between the two methods. The greatest impact was found on measurement of the maximal AS for which the limits of agreement was $[-9 \% ; 11 \%]$ for $\mathrm{T} 1$ and $[-11 \%$; $13.50 \%$ ] for Fat.

Point-to-surface errors from this study were comparable to previously published values [27-29]. Sudhoff [29] reported 6\% error in muscle volume when compared to a reference object (based on 10 volunteers and 2 operators). Using CT images, Jolivet et al. [28] reported errors (two standard deviations around the mean volume) of 5-12\% in a reproducibility analysis of the hip muscles of 30 subjects ( 3 operators). In the current study, which includes only two volunteers, errors were on average, less than $5 \%$ for all parameters. Of note, the current protocol used pre-defined slices to identify the muscle insertion.

The variation of reproducibility among the different muscles may be explained by several reasons. First, independent of the image considered, the distinction between muscles was not always straightforward (e.g. gluteus minimus versus gluteus medius or vastus medialis versus vastus intermedius) - a 


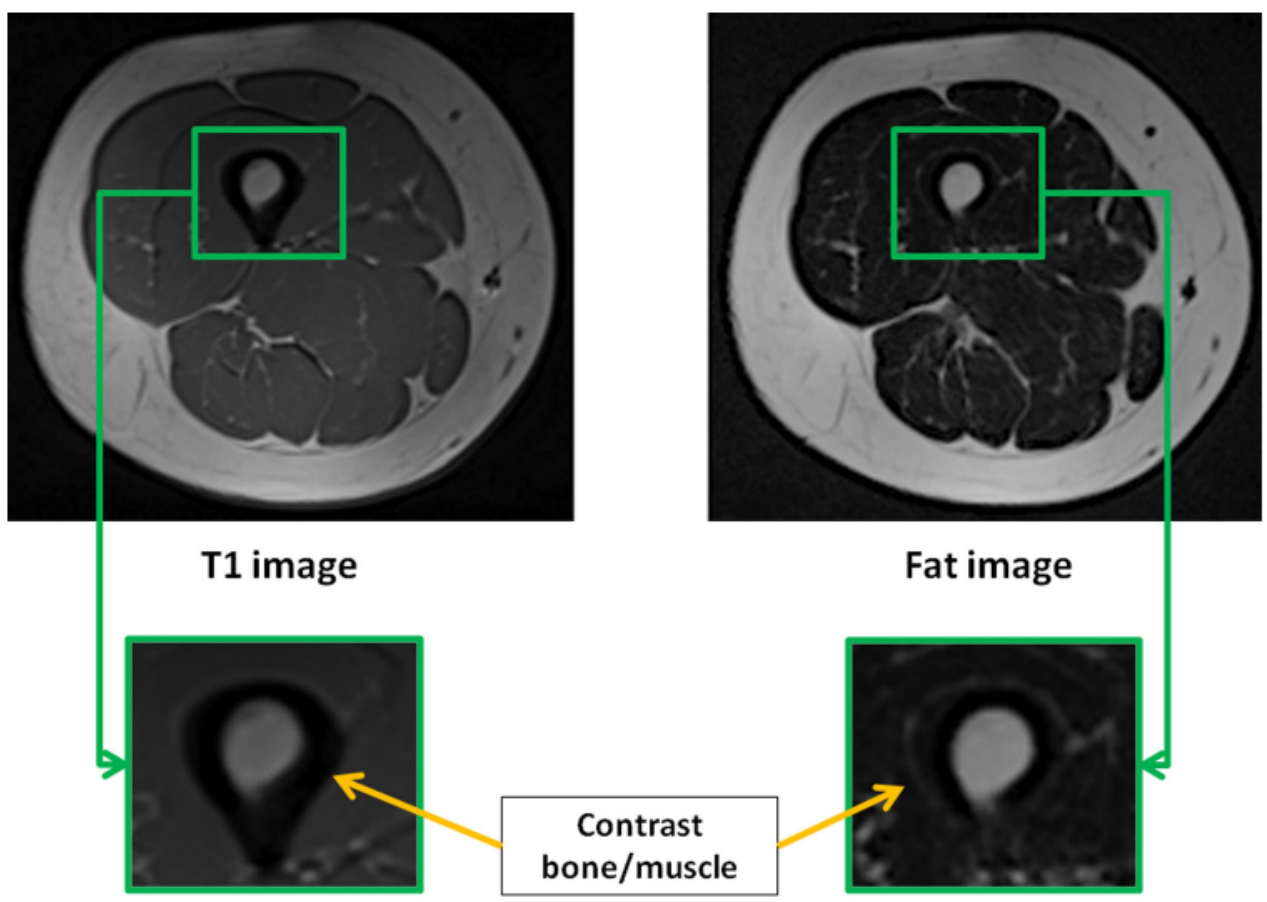

Fig. 4. Contrast between bone/muscle in $\mathrm{T} 1$ and Fat images.

difficulty previously reported by other authors [29]. Second, MRI acquisitions were acquired in free breathing, which led to motion artifacts in the abdominal region, that may compromise accuracy of segmentation of the abdominal muscles (e.g. the rectus abdominus and obliquus).

In conclusion, this study presents a validation of a protocol to characterize muscle geometry and quantification of the fat infiltration with MRI. The combination of the Muscl'X software with the DPSO method and the three point Dixon method demonstrated good agreement, a reproducibility of less than $5 \%$ and led to a substantial gain in reconstruction time. The imaging protocol included in this work is broadly available for clinical scans and has the potential to assess muscular differences between patients and thus the ability to generate patient-specific musculoskeletal models.

\section{References}

[1] Cho KJ, Suk SI, Park SR, et al. Risk factors of sagittal decompensation after long posterior instrumentation and fusion for degenerative lumbar scoliosis. Spine (Phila Pa 1976) 2010;35:1595-601.

[2] Glassman SD, Berven S, Bridwell K, et al. Correlation of radiographic parameters and clinical symptoms in adult scoliosis. Spine (Phila Pa 1976) 2005;30:682-8.

[3] Glassman SD, Bridwell K, Dimar JR, et al. The impact of positive sagitta balance in adult spinal deformity. Spine (Phila Pa 1976) 2005;30:2024-9.

[4] Lafage V, Schwab F, Patel A, et al. Pelvic tilt and truncal inclination: two key radiographic parameters in the setting of adults with spinal deformity. Spine (Phila Pa 1976) 2009;34:E599-606.

[5] Gille O, Jolivet E, Dousset V, et al. Erector spinae muscle changes on magnetic resonance imaging following lumbar surgery through a posterior approach. Spine (Phila Pa 1976) 2007;32:1236-41.

[6] Kawaguchi Y, Matsui H, Tsuji H. Back muscle injury after posterior lumbar spine surgery. Part 2: histologic and histochemical analyses in humans. Spine 1994;19:2598-602.
[7] Kawaguchi Y, Matsui H, Tsuji H. Back muscle injury after posterior lumbar spine surgery. A histologic and enzymatic analysis. Spine 1996;21:941-4.

[8] Taylor H, McGregor AH, Medhi-Zadeh S, et al. The impact of self-retaining retractors on the paraspinal muscles during posterior spinal surgery. Spine 2002;27:2758-62.

[9] Weber BR, Grob D, Dvorak J, et al. Posterior surgical approach to the lumbar spine and its effect on the multifidus muscle. Spine 1997;22:1765-72.

[10] Flicker PL, Fleckenstein JL, Ferry K, et al. Lumbar muscle usage in chronic low back pain. Magnetic resonance image evaluation. Spine 1993;18:582-6.

[11] Gejo R, Matsui H, Kawaguchi Y, et al. Serial changes in trunk muscle performance after posterior lumbar surgery. Spine 1999;24:1023-8.

[12] Kim DY, Lee SH, Chung SK, et al. Comparison of multifidus muscle atrophy and trunk extension muscle strength: percutaneous versus open pedicle screw fixation. Spine 2005;30:123-9.

[13] Humphrey AR, Nargol AV, Jones AP, et al. The value of electromyography of the lumbar paraspinal muscles in discriminating between chronic-lowback-pain sufferers and normal subjects. Eur Spine J 2005;14:175-84.

[14] Mooney V, Gulick J, Perlman M, et al. Relationships between myoelectric activity, strength, and MRI of lumbar extensor muscles in back pain patients and normal subjects. J Spinal Disord 1997;10:348-56.

[15] Barker KL, Shamley DR, Jackson D. Changes in the cross-sectional area of multifidus and psoas in patients with unilateral back pain: the relationship to pain and disability. Spine 2004;29:E515-9.

[16] Dangaria TR, Naesh O. Changes in cross-sectional area of psoas major muscle in unilateral sciatica caused by disc herniation. Spine 1998;23: 928-31.

[17] Danneels LA, Vanderstraeten GG, Cambier DC, et al. CT imaging of trunk muscles in chronic low back pain patients and healthy control subjects. Eur Spine J 2000;9:266-72.

[18] Hultman G, Nordin M, Saraste H, et al. Body composition, endurance, strength, cross-sectional area, and density of MM erector spinae in men with and without low back pain. J Spinal Disord 1993;6:114-23.

[19] Keller A, Gunderson R, Reikeras O, et al. Reliability of computed tomography measurements of paraspinal muscle cross-sectional area and density in patients with chronic low back pain. Spine 2003;28:1455-60.

[20] Mayer TG, Vanharanta H, Gatchel RJ, et al. Comparison of CT scan muscle measurements and isokinetic trunk strength in postoperative patients. Spine 1989;14:33-6. 
[21] Parkkola R, Rytokoski U, Kormano M. Magnetic resonance imaging of the discs and trunk muscles in patients with chronic low back pain and healthy control subjects. Spine 1993;18:830-6.

[22] Savage RA, Millerchip R, Whitehouse GH, et al. Lumbar muscularity and its relationship with age, occupation and low back pain. Eur J Appl Physiol Occup Physiol 1991;63:265-8.

[23] Storheim K, Holm I, Gunderson R, et al. The effect of comprehensive group training on cross-sectional area, density, and strength of paraspinal muscles in patients sick-listed for subacute low back pain. J Spinal Disord Tech 2003;16:271-9.

[24] Airaksinen O, Herno A, Kaukanen E, et al. Density of lumbar muscles 4 years after decompressive spinal surgery. Eur Spine J 1996;5:193-7.

[25] Salminen JJ, Erkintalo-Tertti MO, Paajanen HE. Magnetic resonance imaging findings of lumbar spine in the young: correlation with leisure time physical activity, spinal mobility, and trunk muscle strength in 15-year-old pupils with or without low-back pain. J Spinal Disord 1993;6:386-91.

[26] Tracy BL, Ivey FM, Jeffrey Metter E, et al. A more efficient magnetic resonance imaging-based strategy for measuring quadriceps muscle volume. Med Sci Sports Exerc 2003;35:425-33.

[27] Jolivet E, Daguet E, Pomero V, et al. Volumic patient-specific reconstruction of muscular system based on a reduced dataset of medical images. Comput Methods Biomech Biomed Engin 2008;11:281-90.

[28] Jolivet $\mathrm{E}$ [PhD report] Biomechanical modelisation of hip and its soft tissue for hip fracture risk. Paris: Laboratory of Biomecanics. ENSAM; 2007. p. 177.

[29] Sudhoff I, de Guise JA, Nordez A, et al. 3D-patient-specific geometry of the muscles involved in knee motion from selected MRI images. Med Biol Eng Comput 2009;47:579-87.

[30] Dixon WT. Simple proton spectroscopic imaging. Radiology 1984;153:189-94.
[31] Glover GH, Schneider E. Three-point Dixon technique for true water/fat decomposition with B0 inhomogeneity correction. Magn Reson Med 1991;18:371-83.

[32] Glover GH. Multipoint Dixon technique for water and fat proton and susceptibility imaging. J Magn Reson Imaging 1991;1:521-30.

[33] Yeung HN, Kormos DW. Separation of true fat and water images by correcting magnetic field inhomogeneity in situ. Radiology 1986;159: 783-6.

[34] Angotti A. Contribution à l'analyse musculaire de patients présentant des pathologies rachidiennes. Paris: Laboratoire de Biomecanique. ENSAM; 2008. p. 55 ,

[35] Tochu F. A contouring program based on dual kriging interpolation. Eng Comput 1993;0:160-77.

[36] Martin Bland J. Statistical methods for assessing agreement between two methods of clinical measurement. Lancet 1986:307-10.

[37] Standardization II Of. Accuracy (trueness and precision) of measurement methods and results - Part 2: basic method for the determination of repeatability and reproducibility of a standard measurement method: 1994, 1994.

[38] Mitton D, Landry C, Veron S, et al. 3D reconstruction method from biplanar radiography using non-stereo corresponding points and elastic deformable meshes. Med Biol Eng Comput 2000;38:133-9.

[39] Pomero V, Lavaste F, Imbert G, et al. A proprioception based regulation model to estimate the trunk muscle forces. Comput Methods Biomech Biomed Engin 2004;7:331-8.

[40] Pomero V, Vital JM, Lavaste F, et al. Muscular modelling: relationship between postural default and spine overloading. Stud Health Technol Inform 2002;88:321-5.

[41] Bley TA, Wieben O, Francois CJ, et al. Fat and water magnetic resonance imaging. J Magn Reson Imaging 2010;31:4-18. 